\title{
STRONGLY NONLINEAR VORTICES IN MAGNETIZED FERROFLUIDS
}

\author{
CRAIG L. RUSSELL ${ }^{1}$, P. J. BLENNERHASSETT ${ }^{2}$ and P. J. STILES ${ }^{1}$
}

(Received 26 May 1997; revised 24 September 1997)

\begin{abstract}
Nonlinear convective roll cells that develop in thin layers of magnetized ferrofluids heated from above are examined in the limit as the wavenumber of the cells becomes large. Weakly nonlinear solutions of the governing equations are extended to solutions that are valid at larger distances above the curves of marginal stability. In this region, a vortex flow develops where the fundamental vortex terms and the correction to the mean are determined simultaneously rather than sequentially. The solution is further extended into the nonlinear region of parameter space where the flow has a core-boundary layer structure characterized by a simple solution in the core and a boundary layer containing all the harmonics of the vortex motion. Numerical solutions of the boundary layer equations are presented and it is shown that the heat transfer across the layer is significantly greater than in the conduction state.
\end{abstract}

\section{Introduction}

Ferrofluids are commercially manufactured colloidal liquids usually formed by suspending small particles of magnetite in a liquid medium such as heptane, kerosene or water. The magnetic susceptibility of a typical ferrofluid lies between those of paramagnetic and ferromagnetic materials and hence, when subjected to an external magnetic field, a ferrofluid becomes magnetized [16]. Owing to their unique properties, ferrofluids have emerged as reliable materials capable of solving complex engineering problems. Nonlinear thermal convection in magnetized ferrofluids is a topic of current technical importance since magnetic forces can be used to create circulation of coolant in small passages where natural convection is either absent or ineffective. Examples of current uses include the cooling of electrical equipment such as loudspeakers as well as many uses as dynamic process and exclusion seals [15].

\footnotetext{
${ }^{1}$ School of Chemistry, Macquarie University, NSW 2109, Australia.

${ }^{2}$ School of Mathematics, University of New South Wales, NSW 2052, Australia.

(C) Australian Mathematical Society, 1998, Serial-fee code 0334-2700/98
} 
Previous theoretical work has defined conditions required for the onset of convection in the presence of a magnetic field $[2,8,24,26]$ but the analysis of flow patterns and heat transfer rates that result in systems operating far from onset conditions has not been studied in detail.

The Navier-Stokes equations of motion of a magnetized ferrofluid contain a magnetic body force term $\mu_{0} \boldsymbol{M} \cdot \nabla \boldsymbol{H}$, where $\mu_{0}$ is the permeability of free space, $\boldsymbol{M}$ the magnetization of the ferrofluid and $\nabla \boldsymbol{H}$ the gradient of the magnetic field $\boldsymbol{H}$. Experiments $[20]$ and theoretical investigations $[2,8,24,26]$ have shown that an horizontal layer of ferrofluid in an externally uniform, vertical magnetostatic field and a vertical temperature gradient is unstable to periodic roll cells. Vortex motion can be induced in the ferrofluid layer when the magnetic Rayleigh number, which measures the ratio of the magnetic body force to the dissipative forces and is an analogue of the more familiar gravitational Rayleigh number, exceeds its critical value. Of particular interest in the case of magnetized ferrofluids is that this motion can occur when the ferrofluid is heated from above, in which case gravity is stabilizing and only the magnetic body force can cause instability.

Previous analyses of the instability of a ferrofluid layer $[2,8,26]$ have been confined to linear and weakly nonlinear treatments in which disturbances to the basic state are small. The aim of this paper is to demonstrate that 'strongly nonlinear' vortices are possible in magnetized ferrofluids when the wavenumber of the disturbance is large. Similar vortex structures have been found in other hydrodynamic problems, namely the stability of boundary layer flow over a curved surface [10], Taylor vortices between rotating concentric cylinders [5] and Rayleigh-Bénard convection in non-magnetic Newtonian fluids [1]. In the Taylor and Rayleigh-Bénard problems, these large wavenumber strongly nonlinear vortices are a natural generalization of the weakly nonlinear solutions valid near the linear-theory marginal curve.

We show here that for large wavenumbers, 'strongly nonlinear' vortex structures can be found as asymptotic solutions of the governing equations in a manner similar to that used for the Rayleigh-Bénard problem [1]. Unlike the Rayleigh-Bénard problem however, a unique curve of marginal stability does not exist. The description 'strongly nonlinear' vortex motion has never been rigorously defined but has come to be applied to two types of flow. First, to flows in which, due to the presence of vortices, the mean flow profile is dramatically altered from its unperturbed state. This is the case in boundary layer flows over curved surfaces [9]. Second, to flows in which the shape of the fundamental mode in the Fourier representation is very different to that obtained from linear theory. This is the case in the Rayleigh-Bénard and magnetized ferrofiuid problems in which the basic conduction (mean) state is the leading order term in the solution for the temperature profile.

To obtain these strongly nonlinear vortex structures we consider the case where the magnetic field is sufficiently weak to ensure that the magnetization varies linearly 
with the field strength and that the magnetic susceptibility of the ferrofluid is isotropic. To describe the vortices that develop in the ferrofluid layer we require not only the Navier-Stokes equations for linear momentum, the temperature equation for energy conservation and the continuity equation, but also the Maxwell equations $\nabla \cdot B=0$ and $\nabla \times \boldsymbol{H}=\mathbf{0}$ for a non-conducting fluid with no displacement currents (note that $\boldsymbol{B}$, the magnetic induction, is equal to $\mu_{0}(\boldsymbol{H}+\boldsymbol{M})$ ). Also, two sets of boundary conditions are considered. The first, referred to as 'free-free' conditions, are an idealization and correspond to zero tangential stress at the boundaries and are chosen to allow simple exact solutions of the governing equations. The second, referred to as 'rigidrigid' conditions, are more physically realistic and correspond to no-slip conditions being imposed at the boundaries between the ferrofluid layer and magnetic polepieces. It is subsequently shown that as the wavenumber of the vortices (convection cells) increases, the solution of the linear stability problem with rigid-rigid boundary conditions asymptotically approaches the solution of the linear stability problem with free-free boundary conditions.

The rest of this paper is organized as follows. Section 2 outlines a mathematical model of the ferrofluid layer and the equations governing the onset of convection. Section 3 summarizes the weakly nonlinear results when the instability parameter, the magnetic Rayleigh number, is close to the linear stability marginal curve. The large wavenumber limit (for a constant value of the gravitational Rayleigh number) of these results is obtained and thence used in Section 4 to derive an intermediate structure between those of the weakly and strongly nonlinear treatments. In Section 5 the solution is extended into the strongly nonlinear region where a numerical solution of the boundary layer equations is required and some results for both the free-free and rigid-rigid conditions are presented.

\section{The governing equations}

Consider an infinite horizontal layer of an incompressible ferrofluid confined between two isothermal plates a distance $d$ apart. Let the temperatures of the upper and lower plates be $T_{U}$ and $T_{L}$ respectively and use a Cartesian coordinate system $(x, y, z)$ with the $y$-axis normal to the bounding surfaces of the fluid and gravity acting in the negative $y$-direction. Distances in the three coordinate directions are measured by $x d, y d$ and $z d$ and the fluid boundaries are the planes $y=0$ and 1 . Onto the layer of ferrofluid impose a spatially uniform magnetostatic field in the vertical direction. For sufficiently small temperature differences between the plates we assume the ferrofluid satisfies the Oberbeck-Boussinesq approximation, in which variations in density are considered only in the gravitational body force, and hence that the coefficient of thermal expansion $\alpha$, the thermal diffusivity $\kappa$ and the shear viscosity $\eta$ are 
constants. In doing so we are neglecting dissipative magnetic forces responsible for magnetoviscosity $[21,23]$ and are ignoring the effects of magnetoviscosity due to the spin angular velocity of the colloidal particles, an effect that has been investigated by Stiles and Kagan [24]. Also, we consider only short time scale instabilities, thereby ignoring the Soret effect which describes solute diffusion up or down a temperature gradient $[4,12]$ and magnetodiffusion, a process by which the magnetic dipoles of the colloidal particles are attracted towards the region where the magnetic field strength is greatest [3]. In each of these cases the temporal dependence of the particle flux perturbs both steady conduction and convection. However, a simple analysis suggests that these diffusive processes might lead to concentration changes of no more than a few percent over a period of several days. Since measurements of heat transfer can be performed on a much shorter time-scale it is reasonable to omit this complication. The influence of particle diffusion over long time scales has been examined by Stiles $e t$ $a l$. [25]. Under these assumptions the fluid density $\rho$ depends linearly on variations in temperature $T$ as $\rho_{0}\left[1-\alpha\left(T-T_{0}\right)\right]$, where $\rho_{0}$ is a reference density of the fluid at temperature $T_{0}$, the average of the temperatures at the top and bottom plates. Note that we have also assumed that the fluid properties $\alpha, \kappa, \eta$ and $\rho$ are all independent of the applied magnetic field.

The local magnetization is considered to be a function of $\boldsymbol{H}$ and $T$ in the form $\chi(T) H$, where $\chi$ is the magnetic susceptibility. This expression applies quite generally when the magnetic field is weak and we assume that $\chi$ can be written in a form similar to the density as $\chi_{0}+\left.(d \chi / d T)\right|_{T_{0}}\left(T-T_{0}\right)$. Note that, since the applied field is static and the ferrofluid is not electrically conducting, the electric field vector vanishes in this problem. In addition, the relaxation time of the magnetization of a ferrofluid is so small that the dynamics of it can be disregarded in the analysis of hydrodynamic phenomena. This is known as the quasi-stationary assumption of the magnetization $[16,22]$. The average temperature $T_{0}$ and the temperature gradient $\beta$ are given by $\left(T_{U}+T_{L}\right) / 2$ and $\left(T_{L}-T_{U}\right) / d$ respectively, so that $\beta<0$ when the fluid is heated from above.

In the basic conduction state the temperature distribution $\bar{T}(y)$ is $T_{0}-\beta d\left(y-\frac{1}{2}\right)$ and since the magnetic induction outside the fluid layer is vertical, continuity of the vertical component of $\boldsymbol{B}$ and the horizontal components of $\boldsymbol{H}$ across the fluid-plate interface ensures that $\boldsymbol{H}=\bar{H}(y) \boldsymbol{j}$ and $\boldsymbol{M}=\bar{M}(y) j$ in the fluid layer, where $j$ is a unit vector in the positive $y$-direction and

$$
\begin{gathered}
\bar{H}(y)=H_{0}-\frac{K \beta d}{1+\chi_{0}}\left(y-\frac{1}{2}\right), \quad \bar{M}(y)=M_{0}+\frac{K \beta d}{1+\chi_{0}}\left(y-\frac{1}{2}\right), \\
H_{0}=\bar{H}\left(\frac{1}{2}\right), \quad M_{0}=\chi_{0} H_{0}
\end{gathered}
$$

and the pyromagnetic coefficient $K$ is defined by $-\left.H_{0}(d \chi / d T)\right|_{T_{0}}$. Here we have used the magnetic analogue of the Boussinesq approximation, that is, changes in $\chi$ with 
temperature have been ignored except where multiplied by the average field strength $H_{0}$. In the absence of a temperature gradient across the fluid layer, the magnetic induction has the value $\mu_{0}\left(M_{0}+H_{0}\right)$ while $M_{0}+H_{0}=\mu_{r} H_{e}$, where $H_{e}$ is the field strength in the bounding material and $\mu_{r}$ is its relative permeability.

When instability occurs, a non-dimensional temperature perturbation $\theta$ results in the temperature field $\bar{T}+\beta d \theta$, while the Maxwell equation $\nabla \times \boldsymbol{H}=0$ allows $\boldsymbol{H}$ to be written in terms of a potential function $\phi$ as

$$
\boldsymbol{H}=\bar{H}(y) \boldsymbol{j}+\frac{K \beta d}{1+\chi_{0}} \nabla \phi,
$$

so that

$$
M=[\bar{M}(y)-K \beta d \theta] j+\frac{\chi_{0} K \beta d}{1+\chi_{0}} \nabla \phi .
$$

We consider only two-dimensional velocity fields and introduce the non-dimensional velocity $\boldsymbol{u}$ and its components $(0, v(y, z, t), w(y, z, t))$ through $\kappa \boldsymbol{u} / d$. With $d^{2} t / \kappa$ as the time variable, the non-dimensional governing equations for incompressible flow are

$$
\begin{gathered}
\nabla \cdot u=0, \\
\nabla^{2} \phi-\theta_{y}=0, \\
-\frac{\partial \theta}{\partial t}+\nabla^{2} \theta+v=u \cdot \nabla \theta, \\
-\operatorname{Pr}^{-1} \frac{\partial u}{\partial t}+\nabla^{2} u+\left[(R+N) \theta-N \theta_{y}\right] j-\nabla p=\operatorname{Pr}^{-1}(u \cdot \nabla) u \\
+N \theta \nabla \phi_{y},
\end{gathered}
$$

where $\nabla^{2}$ is the two-dimensional Laplacian in the $y z$-plane, $p$ is a suitably scaled effective pressure and $P r, R$ and $N$ are the Prandtl number, gravitational Rayleigh number and magnetic Rayleigh number respectively, with definitions

$$
\operatorname{Pr}=\eta /\left(\rho_{0} \kappa\right), \quad R=\frac{\rho_{0} g \alpha \beta d^{4}}{\eta \kappa} \quad \text { and } \quad N=\frac{\mu_{0} K^{2} \beta^{2} d^{4}}{\eta \kappa\left(1+\chi_{0}\right)} .
$$

Equations (2.1) are those used by Stiles et al. [26] and are similar to those examined by Finlayson [8]. It is also worth pointing out that these equations are parametrically the same as those used by Blennerhassett et al. [2] for the case where the applied magnetic field is strong $\left(H_{e} \rightarrow \infty\right)$ and the ferrofluid is magnetically saturated (their definition of $N$, however, does not contain the term $1+\chi_{0}$ ). In this case, the value of the magnetization is a function of the fluid temperature only and the expression 
used for $M$ is $\left[M_{0}-K\left(T-T_{0}\right)\right](H / H)$, where $K$ is the strong-field pyromagnetic coefficient and $M_{0}$ the saturation magnetization at temperature $T_{0}$. The remainder of the analysis in this paper therefore applies in both the weak and strong field cases.

To complete the specification of the problem, boundary conditions must be imposed at $y=0$ and 1 . Since the bounding surfaces are maintained at the constant temperatures $T_{L}$ and $T_{U}$, the temperature perturbation $\theta$ must vanish there. For the free-free case, $v$ and $w_{y}$ vanish at the boundaries and we impose the condition that $\phi_{y}$ must also vanish at the boundaries. These conditions have previously been used by Finlayson [8]. For the rigid-rigid case, the velocity boundary conditions are that $v$ and $w$ must vanish while for the magnetic potential function we assume the ferrofluid layer is bounded by magnetic pole-pieces in which the ratio of the magnetic permeability of each pole-piece to that of the ferrofluid is very large. The condition on the field $\boldsymbol{H}$ is therefore the same as for an electric field at the surface of a perfect conductor and hence $\phi$ must vanish on the boundaries (for details see Russell [17]). So, the boundary conditions can be stated as

$$
v=w_{y}=\theta=\phi_{y}=0 \quad \text { or } \quad v=w=\theta=\phi_{y}=0 \quad \text { at } \quad y=0,1 .
$$

We should point out that the equations and boundary conditions describing convection in polarized, dielectric liquids [27] are identical to those presented here and hence the results obtained are valid for both problems.

\section{Weakly nonlinear solutions}

It is convenient to introduce a streamfunction $\psi(y, z, t)$ such that $v=-\psi_{z}$ and $w=\psi_{y}$. The continuity equation is now satisfied identically and equations (2.1) reduce to

$$
\begin{gathered}
\nabla^{2} \phi-\theta_{y}=0, \\
-\frac{\partial \theta}{\partial t}+\nabla^{2} \theta-\psi_{z}=-\psi_{z} \theta_{y}+\psi_{y} \theta_{z}, \\
-P r^{-1} \frac{\partial}{\partial t}\left(\nabla^{2} \psi\right)+\nabla^{4} \psi-(R+N) \theta_{z}+N \phi_{y z} \\
=P r^{-1}\left(\psi_{y} \nabla^{2} \psi_{z}-\psi_{z} \nabla^{2} \psi_{y}\right)+N\left(\theta_{y} \phi_{y z}-\theta_{z} \phi_{y y}\right),
\end{gathered}
$$

with boundary conditions

$$
\psi=\psi_{y y}=\theta=\phi_{y}=0 \quad \text { or } \quad \psi=\psi_{y}=\theta=\phi_{y}=0 \quad \text { at } \quad y=0,1 .
$$

The boundary condition $\psi=0$, rather than $\psi_{z}=0$, is necessary to prevent a uniform horizontal flow as a solution to (3.1). The marginal stability of the basic state is 
controlled by the parameters $R$ and $N$, both of which provide effective measures of the temperature difference across the fluid layer, and $R$ is proportional to the temperature gradient while $N$ is proportional to the square of the temperature gradient. Note that while $R$ can be positive or negative, $N$ can never be negative. The sign of $R$ indicates which boundary is hotter. When $R>0$, the lower boundary is hotter and both the gravitational buoyancy force and the magnetic body force are destabilizing. When $R<0$, the upper boundary is hotter, the gravitational buoyancy force is stabilizing and only the magnetic body force can produce instability. For a particular value of $R$ a curve of marginal stability, $N_{m}$, can be found.

For a fixed Rayleigh number and magnetic Rayleigh numbers close to the marginal curve, a weakly nonlinear analysis starts with the hypotheses

$$
\begin{aligned}
\psi(y, z, t)= & \varepsilon A(\tau)\left[\Psi(y)+\varepsilon^{2} A^{2}(\tau) \Psi_{31}(y)\right] \sin a z+\varepsilon^{2} A^{2}(\tau)\left[\Psi_{20}(y)\right. \\
& \left.+\Psi_{22}(y) \sin 2 a z\right]+\cdots, \\
\theta(y, z, t)= & \varepsilon A(\tau)\left[T(y)+\varepsilon^{2} A^{2}(\tau) T_{31}(y)\right] \cos a z+\varepsilon^{2} A^{2}(\tau)\left[T_{20}(y)\right. \\
& \left.+T_{22}(y) \cos 2 a z\right]+\cdots, \\
\phi(y, z, t)= & \varepsilon A(\tau)\left[\Phi(y)+\varepsilon^{2} A^{2}(\tau) \Phi_{31}(y)\right] \cos a z+\varepsilon^{2} A^{2}(\tau)\left[\Phi_{20}(y)\right. \\
& \left.+\Phi_{22}(y) \cos 2 a z\right]+\cdots,
\end{aligned}
$$

where $a$ is the horizontal wavenumber, time has been scaled as $\tau=\varepsilon^{2} t$ so that $\tau$ is a slow time variable, chosen so that nonlinear effects and linear growth of the amplitude $A$ occur at the same order, namely $\varepsilon^{3}$, and the magnetic Rayleigh number is expanded as $N_{m}+\varepsilon^{2} N_{2}+\cdots$, for $\varepsilon \rightarrow 0^{+}$. In terms of $\varepsilon$, the constant $N_{2}$ is assumed to be $O(1)$.

The equations governing the terms at $O(\varepsilon)$ in the expansions for $\psi, \theta$ and $\phi$ determine the linear stability problem. When the free-free boundary conditions are used the solutions are

$$
\begin{gathered}
N_{m}(a)=a^{4}+4 \pi^{2} a^{2}+\left(6 \pi^{4}-R\right)+\pi^{2}\left(4 \pi^{4}-R\right) a^{-2}+\pi^{8} a^{-4}, \\
\Psi(y)=-\left(\frac{a^{2}+\pi^{2}}{a}\right) \sin \pi y, \quad T(y)=\sin \pi y, \quad \Phi(y)=-\left(\frac{\pi}{a^{2}+\pi^{2}}\right) \cos \pi y, \\
\Psi_{22}=T_{22}=\Phi_{22}=0, \\
\Psi_{20}=0, \quad T_{20}=-\left(\frac{a^{2}+\pi^{2}}{8 \pi}\right) \sin 2 \pi y, \quad \Phi_{20}=\frac{a^{2}+\pi^{2}}{16 \pi^{2}}(\cos 2 \pi y-1) .
\end{gathered}
$$

The equations for $\Psi_{31}, T_{31}$ and $\Phi_{31}$ are forced versions of those for $\Psi, T$ and $\Phi$ and have a solution only if a certain solvability condition is satisfied. This condition is derived by the usual method of examining adjoint systems and in the free-free case 
leads to an amplitude $A$ given by

$$
A^{2}=\frac{8 N_{2} a^{4}}{\left(a^{2}+\pi^{2}\right)\left[a^{4} N_{m}+\left(a^{2}+\pi^{2}\right)^{4}\right]} .
$$

The dimensionless convective heat transfer across the fluid layer is determined by the Nusselt number $N u$, defined by

$$
N u=1-\left.\frac{a}{2 \pi} \int_{0}^{2 \pi / a} \frac{\partial \theta}{\partial y}\right|_{y=0} d z
$$

and it is convenient to calculate a scaled Nusselt number $N u^{*}=(N u-1) /\left(N / N_{m}-1\right)$. The results above give

$$
N u^{*}=\frac{2 a^{4} N_{m}}{a^{4} N_{m}+\left(a^{2}+\pi^{2}\right)^{4}} .
$$

When the wavenumber is large, the free-free results have the asymptotic forms

$$
\begin{gathered}
N_{m} \sim a^{4}+4 \pi^{2} a^{2}+\cdots, \\
\Psi(y) \sim-a \sin \pi y, \quad T(y) \sim \sin \pi y, \quad \Phi(y) \sim-a^{-2} \pi \cos \pi y, \\
T_{20}(y) \sim-\frac{a^{2}}{8 \pi} \sin 2 \pi y, \quad \Phi_{20} \sim-\frac{a^{2}}{16 \pi^{2}}(\cos 2 \pi y-1), \\
A^{2} \sim 4 N_{2} a^{-6}\left(1-5 \pi^{2} a^{-2}+\cdots\right), \\
N u \sim 1+\varepsilon^{2} N_{2} a^{-4}+\cdots, \quad N u^{*} \sim 1-\frac{1}{2} R a^{-4}+\cdots .
\end{gathered}
$$

In general, when the rigid-rigid boundary conditions are used a numerical solution is required. However, when the wavenumber is large, an asymptotic solution is possible and hence a relationship between $A$ and $N_{2}$ can be obtained. This large- $a$ structure can then be used to extend the solution to stronger convective motion. For $a \rightarrow \infty$, a perturbation solution gives

$$
N_{m}(a)=a^{4}+4 \pi^{2} a^{2}+\cdots,
$$

and the flow develops boundary layers of thickness $O\left(a^{-1}\right)$ adjacent to $y=0$ and 1 . Away from these layers the core solutions for the $O(\varepsilon)$ functions are

$$
\begin{gathered}
\Psi(y)=-a \sin \pi y+O\left(a^{-1}\right), \quad T(y)=\sin \pi y+O\left(a^{-2}\right) \\
\text { and } \Phi(y)=-a^{-2} \pi \cos \pi y+O\left(a^{-4}\right) .
\end{gathered}
$$

We do not need to know the precise structure of the boundary layer solutions as it can be shown [17] that these layers do not contribute to the solvability condition at leading 
order for large $a$. All that is needed is the sizes of the solutions in the wall layers, which are

$$
\Psi=O(1), \quad T=O\left(a^{-1}\right), \quad \Phi=O\left(a^{-2}\right) .
$$

At $O\left(\varepsilon^{2}\right)$, the terms forcing the generation of the second harmonics in the core region are now much weaker and

$$
\Psi=\left\{\begin{array}{l}
O\left(a^{-1}\right) \\
O(1)
\end{array} \quad, \quad T=\left\{\begin{array}{l}
O\left(a^{-2}\right) \\
O\left(a^{-1}\right)
\end{array} \quad, \quad \Phi= \begin{cases}O\left(a^{-4}\right) & \text { when } y=O(1), \\
O\left(a^{-2}\right) & \text { in the wall layers. }\end{cases}\right.\right.
$$

The mean temperature and magnetic potential corrections are found to be

$$
T_{20}=\left\{\begin{array}{l}
-\frac{a^{2}}{8 \pi} \sin 2 \pi y+O(1) \\
-\frac{a^{2}}{4} y+O(a)
\end{array} \quad, \Phi_{20}=\left\{\begin{array}{r}
-\frac{a^{2}}{16 \pi^{2}}(\cos 2 \pi y-1)+O(1) \\
\text { when } y=O(1), \\
-\frac{a^{2}}{8} y^{2}+O(a) \quad \text { in the wall } \\
\text { layers, }
\end{array}\right.\right.
$$

so when the large wavenumber approximations are used, the solvability condition reduces to $A^{2} \sim 4 N_{2} a^{-6}+\cdots$, and $N u^{*}=-A^{2} N_{m} D T_{20}(0) / N_{2} \sim 1$ in agreement with the large wavenumber expansions for the free-free case ((3.2d) and (3.2e)). Figure 1 shows the variation of $N u^{*}$ with $a$ for the rigid-rigid problem with $R=-10^{2}$ and $R=-10^{7}$. In both cases the Prandtl number is 1 . These results were obtained from a numerical solution and clearly show that, as the wavenumber is increased, the heat transfer obtained in the rigid-rigid problem tends to the asymptote of the free-free problem. It should be noted that, for large negative values of $R$, the convergence is very slow in both the free-free and the rigid-rigid cases. In both plots, $a$ increases from the global minimum of the marginal curve and the difference in values of $N u^{*}$ at smaller values of $a$ reflects the influence of $R$ on the heat transfer. Depending on the value of $R, N u^{*}$ increases or decreases to its asymptote as $a$ increases.

To summarize, the large wavenumber weakly nonlinear analysis for the core solutions in both the free-free and the rigid-rigid problems have the asymptotic forms

$$
\begin{aligned}
N_{m}= & \left(a^{4}+4 \pi^{2} a^{2}+\cdots\right)+\varepsilon^{2} N_{2}+\cdots \\
\psi= & -\varepsilon A a \sin \pi y \sin a z+\varepsilon^{2} A^{2} O\left(a^{-1}\right) \sin 2 a z+\cdots, \\
\theta= & \varepsilon A \sin \pi y \cos a z+\varepsilon^{2} A^{2}\left[-\frac{a^{2}}{8 \pi} \sin 2 \pi y+O\left(a^{-2}\right) \cos 2 a z\right]+\cdots, \\
\phi= & -\varepsilon A a^{-2} \pi \cos \pi y \cos a z+\varepsilon^{2} A^{2}\left[\frac{a^{2}}{16 \pi^{2}}(\cos 2 \pi y-1)\right. \\
& \left.+O\left(a^{-4}\right) \cos 2 a z\right]+\cdots
\end{aligned}
$$




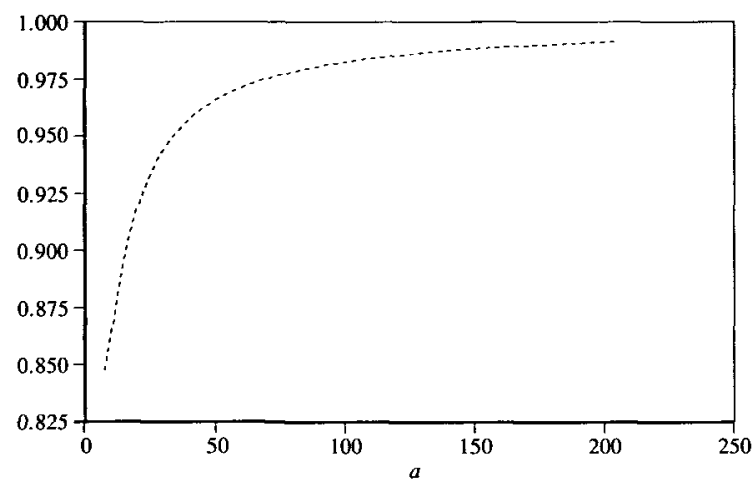

(a) $R=-10^{2}$

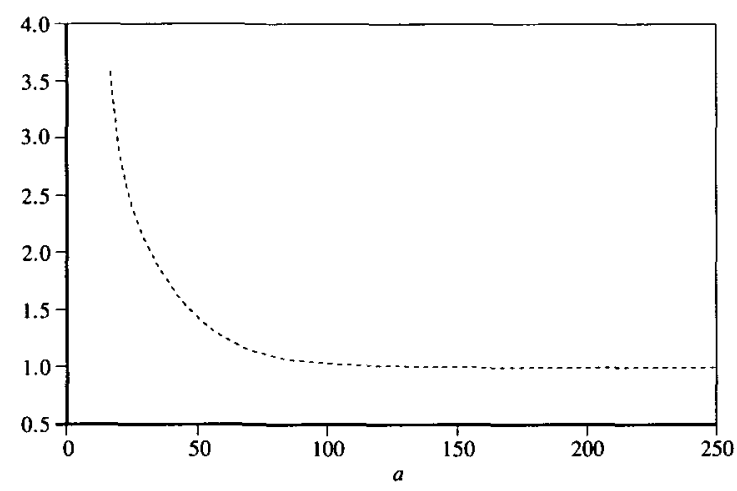

(b) $R=-10^{7}$

FIGURE 1. The scaled Nusselt number $N u^{*}=(N u-1) /\left(N / N_{m}-1\right)$, as a function of the wavenumber $a$, for the rigid-rigid problem with $\operatorname{Pr}=1$ and two values of the Rayleigh number $R$.

where $A=O\left(a^{-3}\right)$ and $R$ is constant. These expansions show that, since the amplitude $A$ is decreasing as the wavenumber gets larger, it is not necessary to enforce the requirement that $\varepsilon$ is small in order to maintain the structure of the solution. In particular, we can make the correction to the mean temperature field the same size as the fundamental, but still keep the temperature perturbations smaller than in the conduction state, by allowing $\varepsilon$ to be as large as $a$. In this case the vertical velocity, $-\psi_{2}$, is $O(1)$, indicating that the convection is much stronger than previously considered. 


\section{Intermediate strength convection}

When $\varepsilon$ is as large as $a$, expansions (3.3) suggest that there is a solution of the form

$$
\begin{aligned}
\psi= & a^{-1}\left(\psi_{10}+a^{-2} \psi_{12}+\cdots\right) \sin a z+a^{-5} \psi_{20} \sin 2 a z+\cdots \\
\theta= & a^{-2}\left(\bar{\theta}_{0}+\bar{\theta}_{2}+\cdots\right)+a^{-2}\left(\theta_{10}+a^{-2} \theta_{12}+\cdots\right) \cos a z \\
& +a^{-6} \theta_{20} \cos 2 a z+\cdots, \\
\phi= & a^{-2}\left(\bar{\phi}_{0}+\bar{\phi}_{2}+\cdots\right)+a^{-4}\left(\phi_{10}+a^{-2} \phi_{12}+\cdots\right) \cos a z \\
& +a^{-8} \phi_{20} \cos 2 a z+\cdots,
\end{aligned}
$$

with

$$
N=a^{4}+N_{2} a^{2}+\cdots \quad \text { as } a \rightarrow \infty,
$$

and where the $\psi_{i j}, \bar{\theta}_{i}, \theta_{i j}, \bar{\phi}_{i}$ and $\phi_{i j}$ are functions of $y$ only. Since $N_{2}$ is $O(1)$ with respect to $a$, a solution of this form describes the convection pattern at greater distances above the marginal curve than the weakly nonlinear theory. These expansions are suitable for the whole flow domain if free-free boundary conditions are used but only for the core region if rigid-rigid boundary conditions are applied. In the latter case, expansions appropriate for the wall layers are required. The problem described here is very similar to the Rayleigh-Bénard problem examined by Blennerhassett and Bassom [1].

The substitution of expansions (4.1) into (3.1) and the comparison of like powers of $a$ and trigonometric terms, leads to the following equations for the unknown functions in (4.1). At leading order in $a$, the vortex terms, that is, terms proportional to $\cos a z$ or $\sin a z$, give

$$
\phi_{10}=-D \theta_{10}, \quad \psi_{10}=-\theta_{10},
$$

where $D$ denotes differentiation with respect to $y$, whilst the mean parts of the leading order Maxwell and energy equations give

$$
D^{2} \bar{\phi}_{0}=D \bar{\theta}_{0}, \quad D^{2} \bar{\theta}_{0}=-\frac{1}{2}\left(\psi_{10} D \theta_{10}+\theta_{10} D \psi_{10}\right) .
$$

At next order in $a^{2}$, the vortex components of (3.1) give

$$
\begin{gathered}
\phi_{12}=D^{2} \phi_{10}-D \theta_{12}, \quad D^{2} \theta_{10}-\theta_{12}-\psi_{12}=-\psi_{10} D \bar{\theta}_{0}, \\
\psi_{12}-2 D^{2} \psi_{10}+N_{2} \theta_{10}+\theta_{12}-D \phi_{10}=\theta_{10} D^{2} \bar{\phi}_{0} .
\end{gathered}
$$

The substitution of (4.2b) into (4.2d) and the consistency of (4.3b) and (4.3c) leads to the following equations for the perturbation $\bar{\theta}_{0}$ to the mean temperature and the 
fundamental $\theta_{10}$,

$$
\begin{gathered}
D^{2} \bar{\theta}_{0}=\theta_{10} D \theta_{10}, \\
D^{2} \theta_{10}+\frac{1}{4} N_{2} \theta_{10}=\frac{1}{2} \theta_{10} D \bar{\theta}_{0},
\end{gathered}
$$

showing that the fundamental and correction to the mean are generated simultaneously, in contrast to the weakly nonlinear calculation where they are generated sequentially. Both these equations are second order and hence we can impose boundary conditions on $\bar{\theta}_{0}$ and $\theta_{10}$. However, since $\theta_{10}$ has even symmetry and $\bar{\theta}_{0}$ has odd symmetry about $y=1 / 2$, we need only consider the interval $[0,1 / 2]$ and so impose the conditions

$$
\bar{\theta}_{0}(0)=\theta_{10}(0)=\bar{\theta}_{0}\left(\frac{1}{2}\right)=D \theta_{10}\left(\frac{1}{2}\right)=0 .
$$

By using (4.4a-c), the remaining leading order mean and first harmonic terms can be determined from $\bar{\theta}_{0}$ and $\theta_{10}$. It follows, via $(4.2 \mathrm{a}, \mathrm{b})$, that the boundary conditions on $\psi_{10}$ and $\phi_{10}$ for the free-free problem are also satisfied so that no boundary layers are required. In the rigid-rigid problem, however, the boundary conditions on $\psi_{10}$ and $\phi_{10}$ are violated and a boundary layer is required. Boundary conditions on $\bar{\phi}_{0}$ can be accommodated in both cases by solving (4.2c), while the solution of (4.4) is valid in both the free-free and rigid-rigid cases.

Second harmonic vortex terms, proportional to $\cos 2 a z$ or $\sin 2 a z$, can be determined by going to the next order in (3.1) to obtain

$$
\begin{gathered}
\phi_{20}=-\frac{1}{4} D \theta_{20} \\
-4 \theta_{20}-2 \theta_{20}=-\frac{1}{2}\left(\psi_{10} D \theta_{12}+\psi_{12} D \theta_{10}-\theta_{12} D \psi_{10}-\theta_{10} D \psi_{12}\right), \\
16 \psi_{20}+2 \theta_{20}=\frac{1}{2}\left[P r^{-1}\left(D \psi_{10} D^{2} \psi_{10}-\psi_{10} D^{3} \psi_{10}\right)\right. \\
\left.+\theta_{10} D^{2} \phi_{10}-D \theta_{10} D \phi_{10}\right]
\end{gathered}
$$

These equations show that the second harmonics are completely determined by the fundamental vortex terms. A similar procedure can be used for higher vortex harmonics but the expansions (4.1) show that these terms become insignificant in the limit as $a \rightarrow \infty$, and the structure of the flow is described by the mean and the fundamental only.

Equations similar to (4.4) have previously been derived for high wavenumber Rayleigh-Bénard convection [1] and in the investigation of the properties of convection cells in double-diffusive systems $[11,13,14]$. It can be shown that the system (4.4) has an analytic solution in terms of elliptic integrals of the first and second kinds. Such a solution, however, is not particularly useful in the present context, since a simple asymptotic state for large $N_{2}$ emerges.

The linear part of (4.4), namely $D^{2} \theta_{10}+\frac{1}{4} N_{2} \theta_{10}=0$, has eigenvalues at $N_{2}=4(2 n-$ $1)^{2} \pi^{2}$, with the corresponding eigenfunctions $\sin (2 n-1) \pi y$, for $n=1,2,3, \ldots$. 
Hence, the nonlinear equations have a solution when $N_{2} \geq 4 \pi^{2}$. Now, equation (4.4a) can be written as $D^{2} \bar{\theta}_{0}=D\left(\frac{1}{2} \theta_{10}^{2}\right)$, so that

$$
D \bar{\theta}_{0}=\frac{1}{2} \theta_{10}^{2}-\bar{A}_{0}
$$

for some real constant $\bar{A}_{0}$. The value of $\bar{A}_{0}$ is determined by integrating (4.6) over $[0,1 / 2]$ to give

$$
\bar{A}_{0}=\int_{0}^{\frac{1}{2}} \theta_{10}^{2} d y=\frac{1}{2} \int_{0}^{1} \theta_{10}^{2} d y,
$$

by symmetry. For values of $N_{2}$ close to $4 \pi^{2}$, an expansion of the form

$$
\begin{aligned}
N_{2}=4 \pi^{2}+n_{2} \delta+\cdots, & \bar{A}_{0}=\bar{a}_{0} \delta+\cdots, \\
\theta_{10}=\delta^{1 / 2}\left(B_{10}+\delta B_{12}+\cdots\right) \sin \pi y+\cdots, & \bar{\theta}_{0}=\delta C_{0} \sin 2 \pi y+\cdots,
\end{aligned}
$$

where $\delta$ is small, gives $B_{10}^{2}=4 n_{2}=-8 \pi C_{0}=4 \bar{a}_{0}$, which matches with the weakly nonlinear expansions (3.3). For larger values of $N_{2}$, both numerical and analytical solutions indicate the emergence of an asymptotic state given by (see Russell [17] for details)

$$
\bar{\theta}_{0} \sim \frac{1}{2} N_{2}\left[y-\frac{1}{2} \tanh \left(\frac{1}{16} N_{2} y\right)\right], \quad \theta_{10} \sim \pm \frac{1}{4 \sqrt{2}} N_{2} \tanh \left(\frac{1}{16} N_{2} y\right)
$$

on the interval $[0,1 / 2]$. The remaining functions in (4.2) therefore have the asymptotic solutions

$$
\begin{gathered}
\psi_{10} \sim \mp \frac{1}{4 \sqrt{2}} N_{2} \tanh \left(\frac{1}{16} N_{2} y\right), \quad \phi_{10} \sim \mp \frac{1}{64 \sqrt{2}} N_{2}^{2} \operatorname{sech}^{2}\left(\frac{1}{16} N_{2} y\right), \\
\bar{\phi}_{0} \sim \frac{1}{4} N_{2}\left\{y^{2}-\frac{16}{N_{2}} \ln \left[\cosh \left(\frac{1}{16} N_{2} y\right)\right]\right\},
\end{gathered}
$$

while the constant $\bar{A}_{0}$ has the asymptotic behaviour

$$
\overline{A_{0}} \sim \frac{1}{64} N_{2}^{2}-\frac{1}{2} N_{2} .
$$

These solutions show that the strength of the vortex motion increases with increasing $N_{2}$, with boundary layers of thickness $O\left(N_{2}^{-1}\right)$ appearing. Thus, when $N_{2}=O(a)$ the $y$-derivatives will be the same size as the $z$-derivatives and all the harmonics will be excited in the boundary layer. Figures 2 and 3 show profiles of $\theta_{10}(y)$ and $\bar{\theta}_{0}(y)$ respectively, for a range of values of $N_{2}$. These solutions were generated numerically and are in good agreement with the asymptotic results (4.9). This agreement is demonstrated in Table 1 , in which the numerically calculated value of $\bar{A}_{0}$ is compared with the asymptotic prediction (4.9f). 
TABLE 1. Comparison of the value of $\bar{A}_{0}$ as a function of $N_{2}$ from a numerical solution and the asymptotic result (4.9f).

\begin{tabular}{|c|l|c|c|}
\hline$N_{2}$ & $\bar{A}_{0}$ & $\frac{1}{64} N_{2}^{2}-\frac{1}{2} N_{2}$ & $\begin{array}{c}\text { relative } \\
\text { error,\% }\end{array}$ \\
\hline 50 & 11.6427 & 14.0625 & -17.20 \\
100 & 106.206 & 106.250 & -0.04 \\
150 & 276.562 & 276.563 & -0.00036 \\
200 & 525.000 & 525.000 & 0.00 \\
250 & 851.563 & 851.563 & 0.00 \\
300 & 1256.25 & 1256.25 & 0.00 \\
\hline
\end{tabular}

The Nusselt number is given by

$$
N u=1-a^{-2} D \bar{\theta}_{0}(0)+\cdots=1+a^{-2} \bar{A}_{0}+\cdots .
$$

By comparing this with the weakly nonlinear result (3.2e), it is clear that the heat transfer is now much greater even though the conduction state $(N u=1)$ still dominates. When $N_{2}=O(a)$ however, there is the possibility that convective effects will make an $O(1)$ contribution to $N u$.

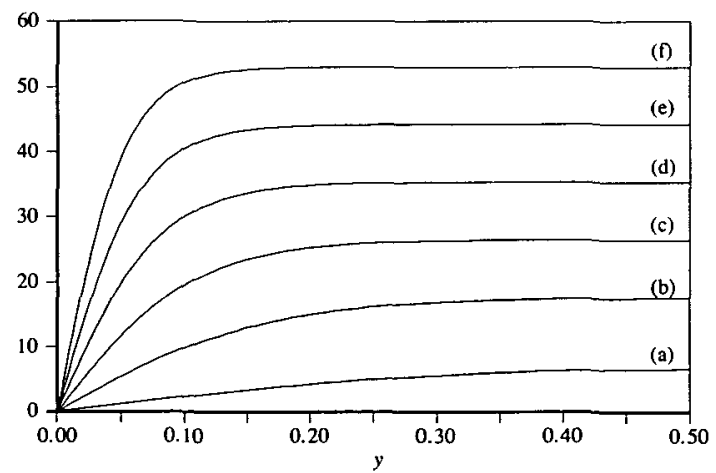

FIGURE 2. Profiles of the temperature fundamental $\theta_{10}(y)$ for a selection of values of the magnetic Rayleigh number coefficient $N_{2}$ (a) $N_{2}=50$; (b) $N_{2}=100$; (c) $N_{2}=150$; (d) $N_{2}=200$; (e) $N_{2}=250$; (f) $\mathrm{N}_{2}=300$.

4.1. Wall layers in the rigid-rigid problem The solutions (4.9) are valid only in the core region away from the walls for the rigid-rigid problem and show that, for 


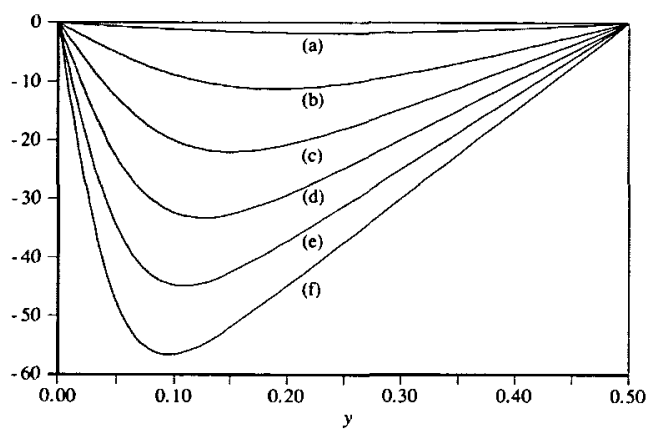

FIGURE 3. Profiles of the mean temperature function $\bar{\theta}_{0}(y)$ for a selection of values of the magnetic Rayleigh number coefficient $N_{2}$ (a) $N_{2}=50$; (b) $N_{2}=100$; (c) $N_{2}=150$; (d) $N_{2}=200$; (e) $N_{2}=250$; (f) $N_{2}=300$.

large $N_{2}, \psi_{10} \rightarrow 0$ with $D \psi_{10}$ finite as $y \rightarrow 0$ and $\phi_{10}$ is finite as $y \rightarrow 0$. Hence, a boundary layer of thickness $O\left(a^{-1}\right)$ is required to satisfy the boundary conditions for $D \psi_{10}$ and $\phi_{0}$. As $y \rightarrow 0, \bar{\theta}_{0} \sim-y, \theta_{10} \sim y, \psi_{10} \sim y, \bar{\phi}_{0} \sim-y^{2}$ and $\phi_{10}$ is constant. Thus, for the layer adjacent to $y=0$, we define the $O(1)$ coordinate $Y=a y$ and use the expansions

$$
\begin{aligned}
\psi & =a^{-2} V_{10} \sin a z+a^{-4} V_{20} \sin 2 a z+\cdots, \\
\theta & =a^{-3} \bar{T}_{0}+a^{-3} T_{10} \cos a z+a^{-5} T_{20} \cos 2 a z+\cdots, \\
\phi & =a^{-4} \bar{P}_{0}+a^{-4} P_{10} \cos a z+a^{-6} P_{20} \cos 2 a z+\cdots,
\end{aligned}
$$

with

$$
N=a^{4}+N_{2} a^{2}+\cdots \quad \text { as } a \rightarrow \infty
$$

where $V_{10}, V_{20}, \bar{T}_{0}, T_{10}, T_{20}, \bar{P}_{0}, P_{10}$ and $P_{20}$ are functions of $Y$. When expansions (4.11) are substituted into (3.1), the governing equations simplify to

$$
\begin{aligned}
\left(D_{Y}^{2}-1\right) P_{10} & =D_{Y} T_{10} \\
\left(D_{Y}^{2}-1\right) T_{10} & =V_{10} \\
\left(D_{Y}^{2}-1\right)^{2} V_{10} & =D_{Y} P_{10}-T_{10} \\
D_{Y}^{2} \bar{P}_{0} & =D_{Y} \bar{T}_{0} \\
D_{Y}^{2} \bar{T}_{0} & =0
\end{aligned}
$$


where $D_{Y}$ denotes differentiation with respect to $Y$. Equations (4.12a-c), governing the vortex terms, reduce to

$$
\left(D_{Y}^{2}-1\right)^{4} T_{10}-T_{10}=0,
$$

while the boundary conditions $V_{10}=D_{Y} V_{10}=T_{10}=P_{10}=0$ at $Y=0$ become

$$
T_{10}=D_{Y}^{2} T_{10}=\left(D_{Y}^{2}-1\right) D_{Y} T_{10}=\left(D_{Y}^{2}-1\right)^{3} D_{Y} T_{10}=0 \quad \text { at } \quad Y=0,
$$

and the other necessary conditions are obtained by matching with the core solutions.

The solution that does not grow exponentially as $Y \rightarrow \infty$ is

$$
\begin{aligned}
T_{10}(Y)= & k_{0}+k_{1} Y+k_{2} \exp \left(-2^{1 / 2} Y\right)+\exp \left[-\left(2^{1 / 4} \cos \pi / 8\right) Y\right] \\
& \left\{k_{3} \sin \left[\left(2^{1 / 4} \sin \pi / 8\right) Y\right]+k_{4} \cos \left[\left(2^{1 / 4} \sin \pi / 8\right) Y\right]\right\} .
\end{aligned}
$$

The constants $k_{1}, k_{2}, k_{3}$ and $k_{4}$ can be chosen in terms of $k_{0}$ so that the required conditions at $Y=0$ are satisfied. For large values of $Y, T_{10} \sim Y$ and matching with the core can only be achieved if $k_{1}=D \theta_{10}(0)=N_{2}^{2} /(64 \sqrt{2})$, thus completely determining $T_{10}(Y)$. The precise values of the remaining $k_{i}$ 's are not required for our purposes.

The solution of $(4.12 \mathrm{e})$ satisfying $\bar{T}_{0}(0)=0$ is $\bar{T}_{0}=c Y$ where $c=\frac{1}{2} N_{2}\left(1-\frac{1}{32} N_{2}\right)=$ $-\bar{A}_{0}$, obtained by matching with the core. Similarly, the solution of (4.12d) satisfying $\bar{P}_{0}(0)=D_{Y} \bar{P}_{0}(0)=0$ is $\bar{P}_{0}=-\frac{1}{2} \bar{A}_{0} Y^{2}$, which also matches with the core. The Nusselt number is therefore

$$
N u=1-a^{-2} D_{Y} \bar{T}_{0}(0)+\cdots=1+a^{-2} \bar{A}_{0}+\cdots,
$$

which is the same as the free-free result (4.10), at leading order.

\section{Strongly nonlinear convection}

From the results of the previous section, it is anticipated that when the magnetic Rayleigh number is $O\left(a^{3}\right)$ above its value at marginal stability the flow field has a core-boundary layer structure. In the core we look for a solution of the form

$$
\begin{aligned}
& \psi=\left(\psi_{10}+a^{-1} \psi_{11}+\cdots\right) \sin a z+a^{-2} \psi_{20} \sin 2 a z+\cdots, \\
& \theta=a^{-1} \bar{\theta}_{1}+a^{-1}\left(\theta_{10}+a^{-1} \theta_{11}+\cdots\right) \cos a z+a^{-3} \theta_{20} \cos 2 a z+\cdots, \\
& \phi=a^{-1} \bar{\phi}_{1}+a^{-3}\left(\phi_{10}+a^{-1} \phi_{11}+\cdots\right) \cos a z+a^{-5} \phi_{20} \cos 2 a z+\cdots,
\end{aligned}
$$

with

$$
N=a^{4}+N_{1} a^{3}+\cdots
$$


as $a \rightarrow \infty$ and where the unknowns $\psi_{i j}, \bar{\theta}_{i}, \theta_{i j}, \bar{\phi}_{i}$ and $\phi_{i j}$ are functions of $y$ only.

As in the previous section, we substitute the expansions (5.1) into (3.1) to obtain, at leading order in powers of $a$,

$$
\phi_{10}=-D \theta_{10}, \quad \psi_{10}=-\theta_{10}
$$

for the vortex components, and

$$
D^{2} \bar{\phi}_{1}=D \bar{\theta}_{1}, \quad 0=-\psi_{10} D \theta_{10}-\theta_{10} D \psi_{10}
$$

for the mean components. Equation (5.2d) shows that $\theta_{10}$ is constant in the core so we can write

$$
\theta_{10}=2 \gamma
$$

for some constant $\gamma$.

Second order vortex terms from the energy and momentum equations give

$$
\theta_{11}+\psi_{11}=\psi_{10} D \bar{\theta}_{1}, \quad \psi_{11}+\theta_{11}+N_{1} \theta_{10}-D \phi_{10}=\theta_{10} D^{2} \bar{\phi}_{1},
$$

showing that the leading order mean temperature perturbation satisfies

$$
D \bar{\theta}_{1}=\frac{1}{2} N_{1} \text {. }
$$

This equation is first order, so boundary conditions at both $y=0$ and 1 cannot be imposed. Owing to the symmetry in the equations and boundary conditions, however, the mean temperature perturbation must be an odd function about $y=1 / 2$. The required solution of $(5.4)$ is therefore

$$
\bar{\theta}_{1}=\frac{1}{2} N_{1}\left(y-\frac{1}{2}\right)
$$

The numerical solutions (Figures 2 and 3), and the large $N_{2}$ asymptotic solution of equations (4.4), also suggest this solution.

The solutions (5.3) and (5.5) do not satisfy the boundary conditions and so boundary layers are required adjacent to $y=0$ and 1 . These layers must be of $O\left(a^{-1}\right)$ thickness for the $y$-derivatives in the governing equations to be at least as large as the $z$ derivatives. Also, to match with the core solutions, $\theta$ must be $O\left(a^{-1}\right)$ and $\psi$ must be $O(1)$ in these layers, while $\phi$ must be $O\left(a^{-2}\right)$ to balance with $\theta$ in the Maxwell equation (3.1a). With these sizes for the perturbation functions it is clear that all the harmonics of the vortex are important in the layers, since the nonlinear interactions in the governing equations generate all harmonics at the same order in $a$.

For the layer adjacent to $y=0$, we define the $O(1)$ co-ordinates $Y=a y$ and $Z=a z$ and expand the perturbation functions in the form

$$
\psi=V(Y, Z), \quad \theta=a^{-1} T(Y, Z), \quad \phi=a^{-2} \Phi(Y, Z) .
$$


The substitution of (5.1d) and (5.6) into (3.1) gives the boundary layer equations, at leading order in powers of $a$, as

$$
\begin{gathered}
\Phi_{Y Y}+\Phi_{Z Z}-T_{Y}=0 \\
T_{Y Y}+T_{Z Z}-V_{Z}=-V_{Z} T_{Y}+V_{Y} T_{Z} \\
V_{Y Y Y Y}+2 V_{Y Y Z Z}+V_{Z Z Z Z}-T_{Z}+\Phi_{Y Z} \\
=\operatorname{Pr}^{-1}\left[V_{Y}\left(V_{Y Y Z}+V_{Z Z Z}\right)-V_{Z}\left(V_{Y Y Y}+V_{Y Z Z}\right)\right]+T_{Y} \Phi_{Y Z}-T_{Z} \Phi_{Y Y}
\end{gathered}
$$

where the subscripts $Y$ and $Z$ denote partial differentiation with respect to $Y$ and $Z$.

These equations are effectively the same as the governing equations since all terms have been retained. The necessary boundary conditions are

$$
V=V_{Y Y}=T=\Phi_{Y}=0 \quad \text { or } \quad V=V_{Y}=T=\Phi=0 \quad \text { at } \quad Y=0,
$$

and matching with the core solutions requires

$$
V \sim-2 \gamma \sin Z, \quad V_{Y} \sim 0, \quad T \sim-\frac{1}{4} N_{1}+2 \gamma \cos Z, \quad \Phi \sim-\frac{1}{4} N_{1} Y
$$

as $Y \rightarrow \infty$.

The equations and boundary conditions (5.7) contain two unknown parameters, the strength $2 \gamma$ of the vortex in the core region and the magnetic Rayleigh number coefficient $N_{1}$, and thus define a nonlinear eigenvalue problem which can only be solved numerically. The Nusselt number can be expressed in terms of $\gamma$ by averaging (5.7b). If $T_{0}(Y)$ denotes the mean temperature field $(1 / 2 \pi) \int_{0}^{2 \pi} T(Y, Z) d Z$, then by averaging $(5.7 \mathrm{~b})$ and using the matching conditions $(5.7 \mathrm{~d}-\mathrm{g})$, we obtain the result

$$
\frac{d T_{0}}{d Y}=-2 \gamma^{2}-\frac{1}{2 \pi} \int_{0}^{2 \pi} V_{Z} T d Z=-2 \gamma^{2}-\frac{1}{2 \pi} \int_{0}^{2 \pi} V T_{Z} d Z
$$

Thus, at leading order in $a$, the Nusselt number is given by

$$
N u=1+2 \gamma^{2}
$$

indicating that the heat transfer additional to the conduction state value is now $2 \gamma^{2}$. By comparing this result with (4.10) and (4.15), and since $\gamma$ is an $O(1)$ constant, it is clear that the convection is now much stronger than in the intermediate state and deserves to be labeled as strongly nonlinear. 
5.1. Numerical solution of the boundary layer equations To complete the description of the vortex structure, a numerical solution of the boundary layer problem (5.7) is required. Since the required solution is $2 \pi$ periodic in $Z$, we look for a solution of the form

$$
\begin{aligned}
& T=\sum_{j=-\infty}^{\infty} T_{j}(Y) E^{j}, \quad T_{-j}=T_{j}, \\
& V=-\mathrm{i} \sum_{j=-\infty}^{\infty} V_{j}(Y) E^{j}, \quad V_{-j}=-V_{j}, \quad V_{0} \equiv 0, \\
& \Phi=\sum_{j=-\infty}^{\infty} P_{j}(Y) E^{j}, \quad P_{-j}=P_{j},
\end{aligned}
$$

where $T_{j}(Y), V_{j}(Y)$ and $P_{j}(Y)$ are real functions of $Y$ and $E \equiv \exp (\mathrm{i} Z)$. The Fourier series (5.9) effectively reduce the governing equations (5.7) to a boundary value problem of an infinite system of coupled ordinary differential equations on a semiinfinite domain. In practice, the series (5.9) must be truncated after a finite number of harmonics, say $M$. The resulting finite-dimensional system of nonlinear equations is then solved on a finite computational domain $\left[0, Y_{\mathrm{inf}}\right]$. Substitution of expansions (5.9) into (5.7), gives $8 M+2$ first order equations for the unknown functions. Since the boundary value problem (5.7) contains two free parameters, $8 M+3$ boundary conditions are required. These are

$$
T_{0}=P_{0}=T_{j}=V_{j}=\frac{d^{2} V_{j}}{d Y^{2}}=\frac{d P_{j}}{d Y}=0 \quad \text { at } \quad Y=0
$$

or

$$
T_{0}=P_{0}=T_{j}=V_{j}=\frac{d V_{j}}{d Y}=P_{j}=0 \quad \text { at } \quad Y=0,
$$

for $j=1, \ldots, M$, together with

$$
\begin{gathered}
T_{0}=-\frac{1}{4} N_{1}, \quad T_{1}=\gamma, \quad V_{1}=-\gamma, \quad \frac{d V_{1}}{d Y}=0, \quad P_{1}=0, \\
T_{j}=V_{j}=\frac{d V_{j}}{d Y}=P_{j}=0 \quad \text { for } j=2,3, \ldots, M,
\end{gathered}
$$

at $Y=Y_{\text {inf }}$.

The results presented here were obtained by keeping terms up to and including $E^{11}$ (that is, $M=11$ ) in the series (5.9), with $Y_{\text {inf }}=25$. Clearly the accuracy of the solution can be increased by taking more terms in the Fourier series, but this increases the number of unknowns in the problem and hence the computational time and memory 
TABLE 2. Values of $\gamma$ as a function of $N_{1}$ for a selection of values of $Y_{\text {inf }}$ with the rigid-rigid boundary conditions and $\operatorname{Pr}=1$.

\begin{tabular}{|c|c|c|c|c|}
\hline$N_{1}$ & $Y_{\text {inf }}=25$ & $Y_{\text {inf }}=50$ & $Y_{\text {inf }}=75$ & $Y_{\text {inf }}=100$ \\
\hline 10 & 0.751826 & 0.751826 & 0.751826 & 0.751826 \\
125 & 5.16999 & 5.16999 & 5.16999 & 5.16999 \\
200 & 7.14734 & 7.14735 & 7.14736 & 7.14737 \\
300 & 9.98387 & 9.98379 & 9.98382 & 9.98385 \\
400 & 12.5013 & 12.5008 & 12.5009 & 12.5009 \\
500 & 14.7915 & 14.7903 & 14.7903 & 14.7903 \\
\hline
\end{tabular}

requirements. Also, for the range of values of $N_{1}$ considered, 11 harmonics proved more than enough for six figure accuracy. The value of $Y_{\text {inf }}$ chosen was the smallest that allowed relatively quick convergence of the solution while still providing adequate resolution of the boundary layers that develop adjacent to $Y=0$. Solutions using smaller values of $M$ and larger values of $Y_{\text {inf }}$ were also obtained in order to test the accuracy of the results (Table 2 shows some values of $\gamma$ as a function of $N_{1}$ for several values of $Y_{\text {inf }}$ ). These tests indicated that the results are correct for $N_{1}$ up to 500 .

The results were found using the routines for solving boundary value problems in the AUTO package [6,7]. Using the automatic continuation facility in AUTO, the starting point for the solution in both the free-free and rigid-rigid cases was the analytic solution of the equations for small $N_{1}$, which is effectively the large $N_{2}$ solution of the intermediate strength convection case (equation (4.9)). The computational problem is very similar to the corresponding boundary layer analysis of large wavenumber Rayleigh-Bénard convection [1] except for one important detail. The values of $N_{1}$ for which reliable results could be obtained are much lower here than those for the corresponding parameter, $R_{1}$, in the Rayleigh-Bénard problem. Blennerhassett and Bassom [1] were able to increase $R_{1}$ up to 4000 compared with 500 for $N_{1}$. The reason for this appears to be due to the more severe nonlinearity in equations (5.7) through the terms $T_{Y} \Phi_{Y Z}$ and $T_{Z} \Phi_{Y Y}$ in the momentum equation, a result of the magnetic mechanism. These terms also play a significant role in the success of the analysis of convection above a critical point [18]. At values of $N_{1}$ greater than 500 , in both the free-free and rigid-rigid cases, the formation of thin boundary layers adjacent to $Y=0$ destroys the accuracy of the solution. The beginning of this effect can be seen in Figures 6(d) and 7(d) close to the wall where the wiggles in the streamlines indicate that there are not enough harmonics in the solution to adequately resolve the boundary layer.

The heat transfer at the wall is determined by the mean temperature function $T_{0}(Y)$, profiles of which are shown in Figure 4 for several values of $N_{1}$ with rigid-rigid 
boundary conditions. Only the region $0 \leq Y \leq 5$ is shown so as to demonstrate the structure close to the wall. As $N_{1}$ increases, the slope of $T_{0}$ at $Y=0$ steepens dramatically, thereby increasing the heat transfer. This is further emphasized in Figure 5, which shows the variation of $\gamma$ with $N_{1}$, by noting that $N u=1+2 \gamma^{2}$ (equation (5.8)) and so the Nusselt number is much greater than its value in the conduction, weakly nonlinear and intermediate strength states.

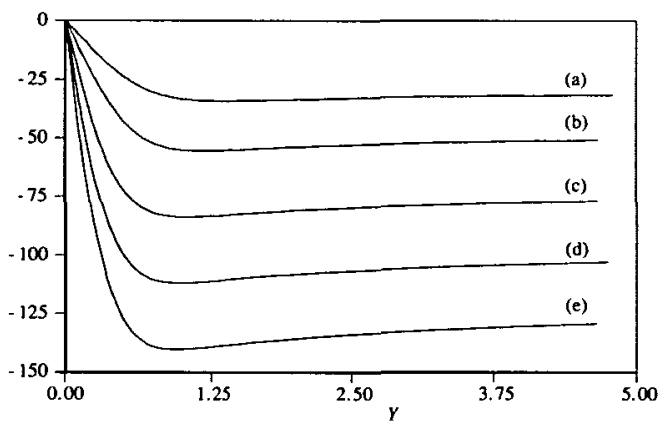

FIGURE 4. Profiles of the mean temperature function $T_{0}(Y)$ with $\operatorname{Pr}=1$, the rigid-rigid boundary conditions and a selection of values of the magnetic Rayleigh number coefficient $N_{1}$. The results were computed on the domain $0 \leq Y \leq 25$, but only the region $0 \leq Y \leq 5$ is shown. (a) $N_{1}=125$; (b) $N_{1}=200 ;$ (c) $N_{1}=300 ;$ (d) $N_{1}=400 ;$ (e) $N_{1}=500$.

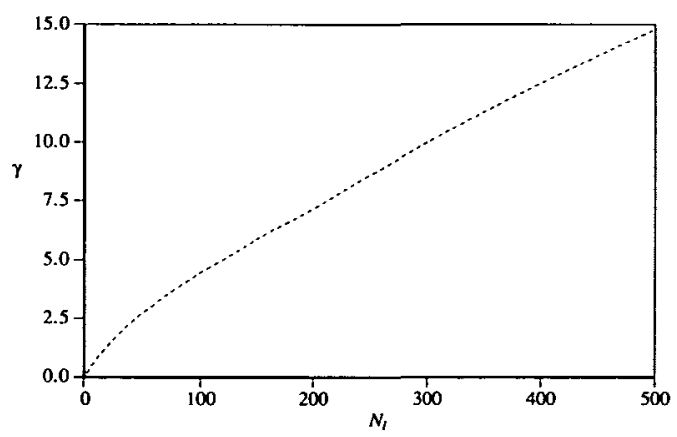

FIGURE 5. The variation of the vortex strength parameter $\gamma$ as a function of $N_{1}$ for the choice of $\operatorname{Pr}=1$ with the rigid-rigid boundary conditions. 


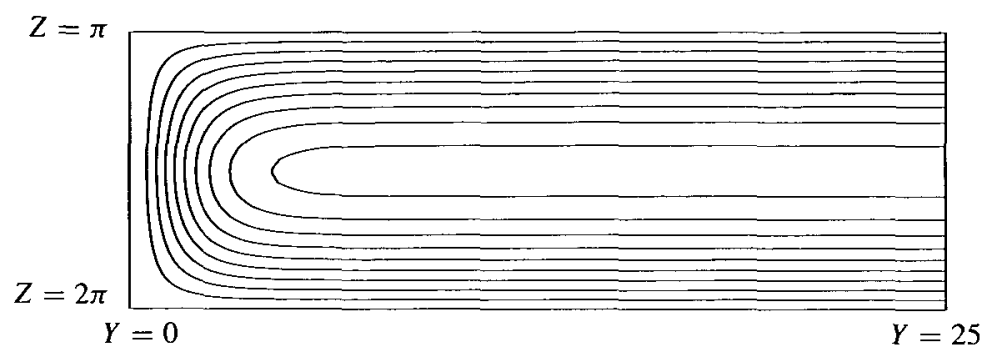

(a) $N_{1}=10, \Delta \psi=0.16, \psi_{\max }=1.44$

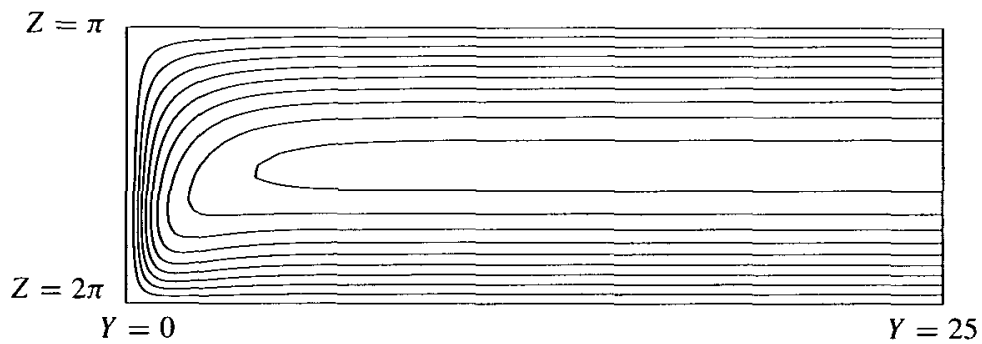

(b) $N_{1}=125, \Delta \psi=1.1, \psi_{\max }=9.90$

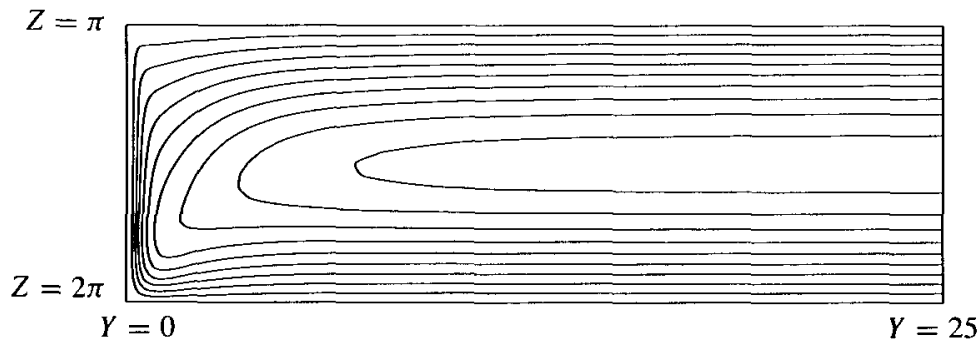

(c) $N_{1}=300, \Delta \psi=2.1, \psi_{\max }=18.9$

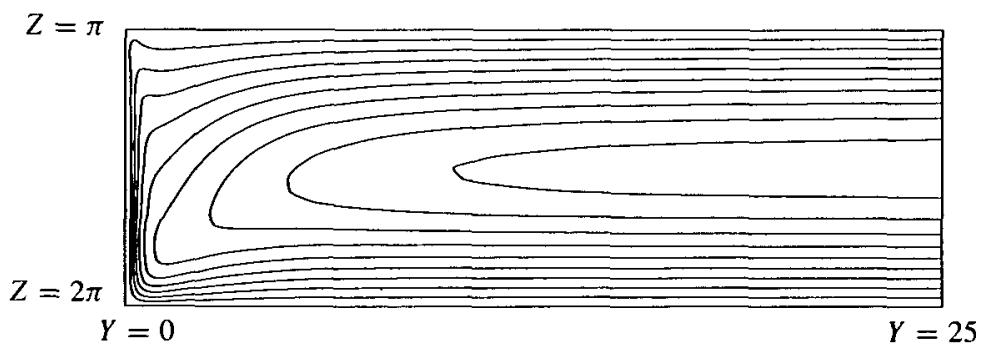

(d) $N_{1}=500, \Delta \psi=3.1, \psi_{\max }=27.9$

FIGURE 6. Lines of constant streamfunction $\psi$ for the solution of equations (5.7) with the rigid-rigid boundary conditions $(5.7 \mathrm{rr})$. The calculations were performed with $M=11$ and $\mathrm{Pr}=1$ on the domain $0 \leq Y \leq 25$. The value of $N_{1}$, the interval $\Delta \psi$ between successive contours and the maximum value $\psi_{\max }$ attained by $\psi$ are shown in each case. The top, bottom and left hand boundaries correspond to $\psi=0$. 


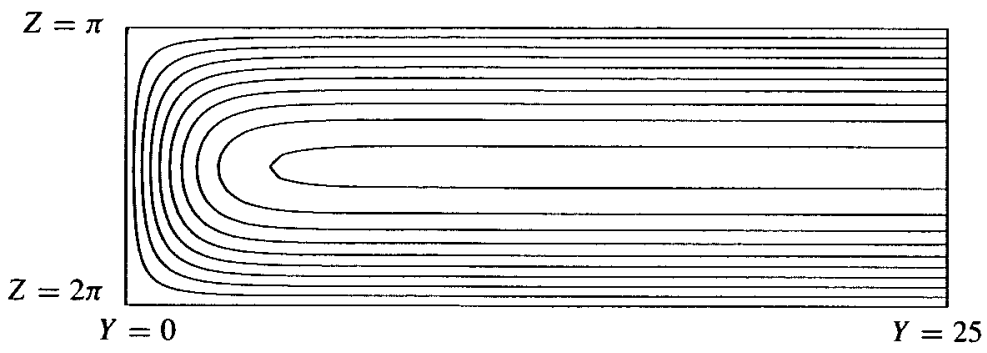

(a) $N_{1}=10, \Delta \psi=0.18, \psi_{\max }=1.62$

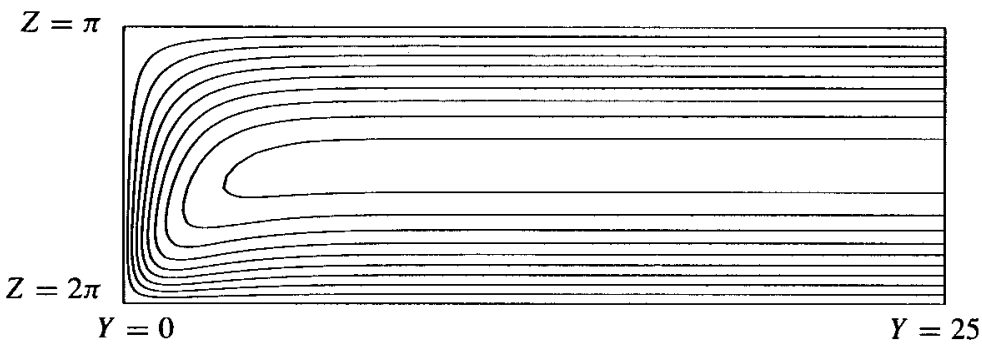

(b) $N_{1}=125, \Delta \psi=1.15, \psi_{\max }=10.35$

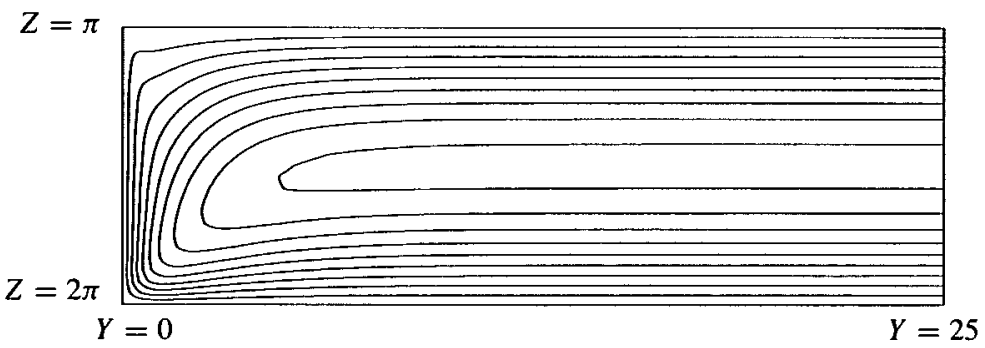

(c) $N_{1}=300, \Delta \psi=2.1, \psi_{\max }=18.9$

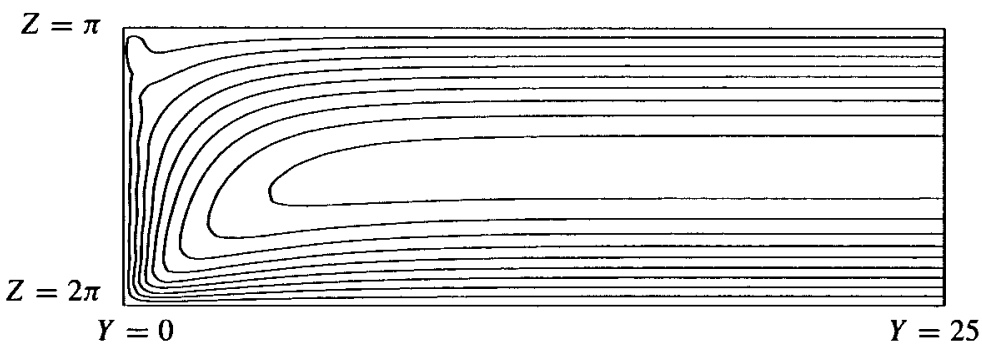

(d) $N_{1}=500, \Delta \psi=2.7, \psi_{\max }=24.3$

FIGURE 7. Lines of constant streamfunction $\psi$ for the solution of equations (5.7) with the free-free boundary conditions (5.7ff). The calculations were performed with $M=11$ and $\mathrm{Pr}=1$ on the domain $0 \leq Y \leq 25$. The value of $N_{1}$, the interval $\Delta \psi$ between successive contours and the maximum value $\psi_{\max }$ attained by $\psi$ are shown in each case. The top, bottom and left hand boundaries correspond to $\psi=0$. 
Figures 6 and 7 show some plots of the streamfunction $\psi$ for the rigid-rigid and free-free boundary conditions respectively, between $Z=\pi$ and $Z=2 \pi$ (the reason for choosing this particular cell rather than $Z=0$ to $\pi$ is so that $\psi$ is positive). The boundaries at $Y=0, Z=\pi$ and $Z=2 \pi$ are the lines $\psi=0$. The vertical velocity is defined by $-\psi_{Z}$ and the horizontal velocity by $\psi_{Y}$, so the diagrams show the fluid moving towards the wall in the top half of each cell (near $Z=\pi$ ), being turned by the wall and then returning to the core in the bottom half of each cell (near $Z=2 \pi$ ). As $N_{1}$ increases a boundary layer clearly develops near $Y=0$ and the flow pattern deviates significantly from the almost symmetrical pattern that occurs when $N_{1}=10$.

\section{Conclusion}

In this paper an asymptotic structure has been found for the vortex pattern which occurs above the large wavenumber end of a marginal curve. In this large wavenumber limit, the flow has a core-boundary layer structure, in which the core region occupies the majority of the flow domain and the boundary layers have $O\left(a^{-1}\right)$ thicknesses near the walls. In the core region only the fundamental and the mean terms determine the flow, while the higher harmonics are negligible, whereas in the boundary layers all the harmonics are important. The solution of the boundary layer equations shows that the heat transfer across the layer in this region of wavenumber-magnetic Rayleigh space is much greater than in the conduction state. The present treatment shows that it is possible to find an asymptotic structure for the vortex pattern in the unstable region of parameter space which is a significant improvement over the weakly nonlinear treatment. The more physically realistic problem of convection occurring above a critical point is more complicated than the problem presented here and is presented in two other papers $[18,19]$. It also demonstrates the similarities between problems involving buoyancy driven instabilities (such as this one and the Rayleigh-Bénard problem) and problems involving centrifugal instability mechanisms (such as the classical Taylor problem) [1].

\section{References}

[1] P. J. Blennerhassett and A. P. Bassom, "Nonlinear high-wavenumber Bénard convection", IMA J. Appl. Math. 52 (1994) 51-77.

[2] P. J. Blennerhassett, F. Lin and P. J. Stiles, "Heat transfer through strongly magnetized ferrofluids", Proc. R. Soc. Lond. A 433 (1991) 165-177.

[3] E. Blums, Y. A. Mikhailov and R. Ozols, Heat and Mass Transfer in MHD Flows (World Scientific, New York, 1987).

[4] S. R. de Groot and P. Mazur, Non-Equilibrium Thermodynamics (Dover, New York, 1984). 
[5] J. P. Denier, "The structure of fully nonlinear Taylor vortices", IMA J. Appl. Math. 49 (1992) 15-33.

[6] E. Doedel, "Auto: A program for the automatic bifurcation analysis of autonomous systems", Cong. Num. 30 (1981) 265-284.

[7] E. Doedel and J. D. Kernevez, "Software for continuation and bifurcation problems", Applied mathematics technical report, Caltech, USA, 1985.

[8] B. A. Finlayson, "Convective instability of ferromagnetic fluids", J. Fluid Mech. 40 (1970) 753-67.

[9] P. Hall, "Görtler vortices in growing boundary layers: the leading edge receptivity problem, linear growth and the nonlinear breakdown stage", Mathematika 37 (1990) 151-89.

[10] P. Hall and W. D. Lakin, "The fully nonlinear development of Görtler vortices in growing boundary layers", Proc. R. Soc. Lond. A 415 (1988) 421-44.

[11] E. Knobloch and M. R. E. Proctor, "Nonlinear periodic convection in double-diffusive systems", J. Fluid Mech. 108 (1981) 291-3l6.

[12] L. D. Landau and E. M. Lifshitz, Fluid Mechanics (Pergamon, Oxford, 1959).

[13] M. R. E. Proctor, "Columnar convection in double-diffusive systems", Cont. Math. 56 (1986) 267-76.

[14] M. R. E. Proctor and J. Y. Holyer, "Planform selection in salt fingers", J. Fluid Mech. 168 (1986) 241-53.

[15] K. Raj and R. Moskowitz, "Commercial applications of ferrofluids", J.Magn. magn. Mater. 85 (1990) 233-45.

[16] R. E. Rosensweig, Ferrohydrodynamics (Cambridge University Press, 1985).

[17] C. L. Russell, "Non-linear thermal convection in magnetised ferrofluids", Ph. D. Thesis, Macquarie University, 1997.

[18] C. L. Russell, P. J. Blennerhassett and P. J. Stiles, "Supercritical analysis of strongly nonlinear vortices in magnetized ferrofluids", Proc. Roy. Soc. Lond. A. (in press).

[19] C. L. Russell, P. J. Blennerhassett and P. J. Stiles, "Weakly nonlinear stability of magnetized ferrofluids at large critical wavenumbers", Applied Mathematics report AMR 97/17, University of NSW, Australia.

[20] L. Schwab, U. Hildebrandt and K. Stierstadt, "Magnetic Bénard convection", J. Magn. magn. Mater: 39 (1983) 113-4.

[21] M. I. Shliomis, "Effective viscosity of magnetic suspensions", Soviet Phys. JETP 34 (1972) 129194.

[22] M. I. Shliomis, "Magnetic fluids", Soviet Phys. Usp. 112 (1974) 153.

[23] P. J. Stiles and M. Kagan, "On the effective viscosity of magnetic colloids in a uniform magnetic field", J. Colloid Interface Sci. 117 (1987) 87-93.

[24] P. J. Stiles and M. Kagan, "Thermoconvective instability of a ferrofluid in a strong magnetic field", J. Colloid Interface Sci. 134 (1990) 435-48.

[25] P. J. Stiles, M. Kagan and P. J. Blennerhassett, "The influence of particle diffusion on the onset of thermoconvective instability magnetized ferrofluids", J. Colloid Interface Sci. 168 (1994) 542-44.

[26] P. J. Stiles, F. Lin and P. J. Blennerhassett, "Heat transfer through weakly magnetized ferrofluids", J. Colloid Interface Sci. 151 (1992) 95-101.

[27] P. J. Stiles, F. Lin and P. J. Blennerhassett, "Convective heat transfer through polarized dielectric liquids", Phys. Fluids A 5 (1993) 3273-9. 